\title{
Dissolution Method Evaluation for Carvedilol Tablets
}

\author{
Josyane Márcia Vasconcelos Alves ${ }^{1,2}$, Livia Deris Prado ${ }^{3}$, and Helvécio Vinícius Antunes Rocha ${ }^{2,3^{*}}$ \\ ${ }^{1}$ Medquímica Indústria Farmacêutica, Juiz de Fora, Brasil. \\ ${ }^{2}$ Programa de Pós-Graduação Profissional em Gestão, Pesquisa e Desenvolvimento na Indústria Farmacêutica, Farmanguinhos, FIOCRUZ, Rio de Janeiro, RJ, \\ Brasil. \\ ${ }^{3}$ Laboratório de Micro e Nanotecnologia, Farmanguinhos, FIOCRUZ, Rio de Janeiro, RJ, Brasil.
}

e-mail: helvecio.far@gmail.com

\section{ABSTRACT}

Carvedilol is an antihypertensive agent with blocking non-selective (selectivity for $\beta 1$ and $\beta 2$ adrenoceptors is moderate) with vasodilating properties conferred on the $\alpha$-receptor blockade. The molecular structure has one chiral center, so the drug exists as two enantiomers. Furthermore, it presents different polymorphs depending on the synthetic route that is used to obtain the drug. Carvedilol is a weak base, practically insoluble in water, acidic solutions, and gastric and intestinal fluids, and is classified as Class II (Biopharmaceutical Classification System). It presents different solubilities in accordance with the $\mathrm{pH}$ of the solvent. In a previous report, it was demonstrated that batches provided by different manufacturers could have different physicochemical properties. The objective of the present study is to evaluate these properties in the formulation of the final tablets and evaluate the best dissolution method. Different media were used to evaluate the impact of raw material characteristics on in vitro release of drug from tablets containing $12.5 \mathrm{mg}$ carvedilol. Pilot batches were obtained by direct compression and wet granulation. Dissolution profiles were compared with a reference drug. Formulations evaluated by wet granulation using carvedilol with a specific particle size $\left(\mathrm{d}_{10} \sim 10\right.$ $\mu \mathrm{m}$ and $d_{90} \sim 95 \mu \mathrm{m}$ ) had similar dissolution profiles to the reference product according to United States Pharmacopeia test 2 and fasted-state simulated intestinal fluid (FaSSIF). The results showed the composition of dissolution medium has a direct impact on the dissolution profile of carvedilol.

KEYWORDS: Carvedilol, dissolution profile, particle size, dissolution medium

\section{INTRODUCTION}

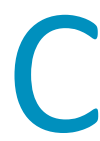
ardiovascular diseases are the cause of many deaths. People worldwide who are affected by numerous cardiovascular problems make use of medicines with the aim of preventing and treating such diseases, including antihypertensive agents to control blood pressure. Carvedilol is an antihypertensive agent with a nonselective blockade for $\alpha$ and $\beta$ receptors, with vasodilatory properties attributed to $\alpha$-receptor blockade (1). This drug is practically insoluble in water and acid solutions, slightly soluble in alcohol, and virtually insoluble in gastric fluid and intestinal fluid $(2,3)$.

Formulation studies performed by Emshanova demonstrate that carvedilol presents low compressibility and does not have good flowability characteristics, and correction of this characteristic is necessary through the use of suitable excipients (4). These studies also concluded that carvedilol is sensitive to light and moisture, and therefore obtaining tablets by direct compression may increase product stability, because in this case there is no contact with liquids as it happens when the wet granulation process is used (5).
After oral administration, carvedilol is rapidly absorbed, reaching maximum plasma concentration in 1 to 2 hours. Absorption is not altered with repeated doses. Feeding slightly alters the rate of absorption of carvedilol without altering the extent of absorption (6).

Carvedilol is a lipophilic drug with a bioavailability after oral administration of approximately $25 \%$ (7). This low bioavailability occurs due to its extensive first-pass metabolism (8). Regarding the structural characteristics, carvedilol has three polymorphs and one hydrate. It has been demonstrated in the literature that the crystalline structures of this drug have different dissolution profiles (9). Thus, polymorphism is a characteristic of carvedilol that may have an impact on its bioavailability.

The development of a generic drug product is guided by pharmaceutical technology challenges, with attention to regulatory requirements, which should be fully met. In Brazil, there is a proper legislation for drug development, which establishes criteria that should compose the registration dossier of a product. 
Tests were carried out to define a generic formulation of $12.5 \mathrm{mg}$ carvedilol tablets. The pilot batches were prepared with this concentration because, according to the United States Food and Drug Administration (FDA) Guidance for Industry on bioequivalence studies; for safety reasons, bioequivalence studies should be conducted with a concentration of $12.5 \mathrm{mg}$, not $25 \mathrm{mg}$, which is the highest dosage for the product (10).

Thus, with a formulation defined for $12.5 \mathrm{mg}$, proportional compositions may be established for other concentrations, and a biowaiver may be required. It should be noted that carvedilol has linear pharmacokinetics, and according to Brazilian legislation, in cases where the other concentrations present proportional formulations, a biowaiver may be required by performing specific tests as recommended by the National Health Surveillance Agency of Brazil (ANVISA), such as the comparative dissolution profile test.

Thus, the objective of this article is to report the paths followed in the development of a generic formulation of carvedilol tablets within the scope of a medium-sized private pharmaceutical company with focus on the dissolution tests used to discriminate the formulations.

\section{MATERIALS AND METHODS}

Materials included: carvedilol (manufacturers A, B, and C), lactose monohydrate spray dried, lactose 200 (DMVFonterra), microcrystalline cellulose 101 and 102 (Blanver); polyvinylpyrrolidone (PVP K-30), crospovidone (ISP/ ASHLAND), colloidal silicon dioxide (Cabot), magnesium stearate (Peter Greven), and partially pregelatinized corn starch (Colorcon). Reference drug product (Coreg) tablets of $12.5 \mathrm{mg}$ were purchased from Roche (lot RJ0440).

\section{Formulation}

Pilot batches were made to obtain a pharmaceutical formulation equivalent to the reference drug product with excipients normally used for producing tablets. Pilot batches qualitative formulations are described in Table 1.

Even by wet granulation or direct compression, the tablets were obtained in a Lawes rotary tablet machine (with 10 punches of $9 \mathrm{~mm}$, concave and smooth). The formulations are described to provide conditions for a deeper discussion about discriminative dissolution conditions.

\section{Hardness Measurement}

The test for determining hardness was performed according to general method 5.1.3.1 of the Brazilian Pharmacopoeia $5^{\text {th }}$ edition (2). The value was determined

Table 1. Qualitative Composition of Pilot Batches

\begin{tabular}{|c|c|c|c|c|c|c|c|}
\hline Raw material & $\begin{array}{c}\text { Batch } \mathrm{Pi}_{1} \\
\text { (DC) }\end{array}$ & $\begin{array}{c}\text { Batch } \mathrm{Pi}_{2} \\
\text { (DC) }\end{array}$ & $\begin{array}{l}\text { Batch } \mathrm{Pi}_{3} \\
\text { (WG) }\end{array}$ & $\begin{array}{c}\text { Batch } \mathrm{Pi}_{4} \\
\text { (WG) }\end{array}$ & $\begin{array}{l}\text { Batch } \mathrm{Pi}_{5} \\
\text { (WG) }\end{array}$ & $\begin{array}{c}\text { Batch } \mathrm{Pi}_{6} \\
\text { (WG) }\end{array}$ & $\begin{array}{c}\text { Batch } \mathrm{Pi}_{7} \\
\text { (WG) }\end{array}$ \\
\hline Carvedilol (A) & $x$ & $x$ & $x$ & $x$ & $x$ & - & - \\
\hline Carvedilol (B) & - & - & - & - & - & $x$ & - \\
\hline Carvedilol (C) & - & - & - & - & - & - & $x$ \\
\hline Lactose monohydrate spray-dried & $x$ & $x$ & $x$ & $x$ & $x$ & $x$ & $x$ \\
\hline Microcrystalline cellulose 102 & $x$ & $x$ & $x$ & $x$ & $x$ & $x$ & $x$ \\
\hline Polyvinylpyrrolidone K-30 & $x$ & $x$ & $x$ & $x$ & $x$ & $x$ & $x$ \\
\hline Crospovidone* & $x$ & $x$ & $x$ & - & $x$ & - & - \\
\hline Colloidal silicon dioxide & $x$ & $x$ & $x$ & $x$ & $x$ & $x$ & $x$ \\
\hline Magnesium stearate & $x$ & $x$ & $x$ & $x$ & $x$ & $x$ & $x$ \\
\hline Partially pregelatinized corn starch & - & $x$ & - & - & - & - & - \\
\hline Lactose 200 & - & - & $x$ & $x$ & $x$ & $x$ & $x$ \\
\hline Microcrystalline cellulose 101 & - & - & $x$ & $x$ & $x$ & $x$ & $x$ \\
\hline Ethanol $96^{\circ} * *$ & - & - & $x$ & $x$ & $x$ & $x$ & $\mathrm{x}$ \\
\hline Purified water** & - & - & $x$ & $x$ & $x$ & $x$ & $x$ \\
\hline
\end{tabular}

Pi, pilot; $D C$, direct compression; WG, wet granulation.

*In pilot batch $\mathrm{Pi}_{3}$, crospovidone intra- and extragranulate were used and in batch $\mathrm{Pi}_{4}$, crospovidone was used only extragranulate.

$* *$ In the wet granulation, a solution of purified water:ethanol $96^{\circ}$ in 1:1 ratio was used. 
on 10 tablets and measured in kgf in a Nova Ética 298-AT (Brazil) hardness tester.

\section{Disintegration Test}

The disintegration test was performed according to general method 5.1.4.1 of the Brazilian Pharmacopoeia $5^{\text {th }}$ edition using six tablets in a tablet disintegration tester Nova Ética 301 AC (2). Disintegration was performed in purified water as immersion liquid at $37 \pm 1{ }^{\circ} \mathrm{C}$ using discs as advocated for plain tablets. It was then given the time required for disintegration of all tablets.

\section{Dissolution Profile Test}

The dissolution profiles of the formulations were evaluated by the quantification of carvedilol in predetermined time intervals $(4,8,12,15$, and 30 minutes). Dissolution equipment was an Eletrolab TDT 08L and spectrophotometer was a Shimadzu UV-1800. The conditions used for determining the profile (dissolution medium, volume, dissolution apparatus, stirring rate) are recommended in the United States Pharmacopoeia (11). Dissolution profiles were also obtained with a biorrelevant medium simulating the empty bowel conditions with the composition proposed by Jantratid and colleagues (12).

There are three dissolution tests (test 1, 2, and 3) presented in the USP monograph for carvedilol. These conditions are shown in Table 2 . In accordance with the monograph of carvedilol, the product must release not less than $80 \%$ ( $Q$ - the amount of dissolved active ingredient specified in the monograph) of the labeled value in 30 minutes (11).

Dissolution profiles of the pilot batches and the reference product were initially obtained using test 2 . This test was chosen to conduct initial evaluation because the medium containing simulated gastric fluid without enzymes is closer to the in vivo conditions compared to test 1 , which is composed only of hydrochloric acid pH 1.45 . The composition of the simulated gastric fluid without enzymes is similar to simulated gastric fluid at preprandial condition proposed by Dressman and Kramer, the latter presenting surfactant in its composition. It should be noted that, although the composition of simulated gastric fluid in the pre-prandial condition has already been revised, this proposal is still closer to the in vivo conditions (13).

After obtaining batches with equivalent dissolution profiles to the reference product in this test, the formulation was kept constant and the manufacturer of the active pharmaceutical ingredient was changed (samples were evaluated for manufacturers A, B, and C). These lots were then subjected to the conditions recommended by USP in test 1 and to the medium containing simulated intestinal fluid for pre-prandial conditions. This medium was chosen considering that the bioequivalence testing for carvedilol should be performed in fasted condition; moreover, the literature reports that for most drugs, the area of maximum absorption following oral administration is the small intestine (14). The conditions such as medium volume, rotation speed, and collection times used in these tests are the same as those recommended for the USP tests 1 and 2 .

\section{RESULTS AND DISCUSSION}

\section{Hardness}

The results of the hardness tests are presented in Table 3. The hardness of the tablets ranged from 3.3 to 10.2 kgf. According to the Brazilian Pharmacopeia $5^{\text {th }}$ edition, the result of this test is just informative (2). For batches in which only the carvedilol manufacturer was changed, keeping the formulation constant, manufacturer $\mathrm{A}\left(\mathrm{Pi}_{4}\right)$ presented hardness results higher than manufacturers $\mathrm{B}$ $\left(\mathrm{Pi}_{6}\right)$ and $\mathrm{C}\left(\mathrm{Pi}_{7}\right)$.

The higher hardness result of $\mathrm{Pi}_{4}$ has certain correlation with the particle size of the sample (15). As it is smaller, there is a larger contact area between the particles,

Table 2. Dissolution Profile Analytical Parameters

\begin{tabular}{|c|c|c|c|}
\hline Conditions & USP Test 1 & USP Test 2 & USP Test 3 (FaSSIF) \\
\hline Agitation system & \multicolumn{3}{|c|}{ Apparatus 2 (paddle) } \\
\hline Speed & \multicolumn{3}{|c|}{$50 \mathrm{rpm}$} \\
\hline Dissolution time & \multicolumn{3}{|c|}{30 minutes } \\
\hline Volume and dissolution medium & $900 \mathrm{~mL} \mathrm{HCl} \mathrm{pH} 1.45$ & $900 \mathrm{~mL}$ simulated gastric fluid without enzymes & $\begin{array}{l}900 \mathrm{~mL} \text { simulated gastric fluid with } \\
\text { pepsine } \mathrm{pH} 1.45\end{array}$ \\
\hline Temperature & \multicolumn{3}{|c|}{$37.0 \pm 0.5^{\circ} \mathrm{C}$} \\
\hline Tolerance & \multicolumn{3}{|c|}{$80 \%(Q)$} \\
\hline Detection method & \multicolumn{2}{|c|}{ UV spectrophotometry $\lambda=285 \mathrm{~nm}$ and $380 \mathrm{~nm}$} & HPLC with ultraviolet detection $\lambda=240 \mathrm{~nm}$ \\
\hline
\end{tabular}

$Q$, amount of dissolved active ingredient specified in the monograph; USP, United States Pharmacopeia; FaSSIF, fasted-state simulated intestinal fluid; UV, ultraviolent; HPLC, high-performance liquid chromatography. 
which makes the adhesive forces greater when subjected to compression. Therefore, it generates a tablet with higher hardness. Lots $\mathrm{Pi}_{6}$ and $\mathrm{Pi}_{7}$ have similar hardness, considering the differences in their particle sizes are also similar. The reference product has a lower hardness compared to the pilot batches produced.

Table 3. Hardness $(n=10)$ and Disintegration Time $(n=6)$ Results

\begin{tabular}{|c|c|c|}
\hline Batch & $\begin{array}{c}\text { Hardness, kgf, } \\
\text { median (range) }\end{array}$ & $\begin{array}{c}\text { Disintegration } \\
\text { Time, mean }\end{array}$ \\
\hline $\mathrm{Pi}_{1}$ (DC) & $6.7(5.9-7.4)$ & $37 \mathrm{~s}$ \\
\hline $\mathrm{Pi}_{\mathbf{2}}$ (DC) & $6.9(6.6-7.4)$ & $1 \mathrm{~min} 32 \mathrm{~s}$ \\
\hline $\mathrm{Pi}_{3}$ (WG; IG and EG) & $6.2(5.4-7.5)$ & $52 \mathrm{~s}$ \\
\hline $\mathrm{Pi}_{4}$ (WG; no disintegrant) & $7.6(5.9-10.2)$ & $1 \mathrm{~min} 2 \mathrm{~s}$ \\
\hline $\mathrm{Pi}_{5}$ (WG) & $7.5(6.3-9.3)$ & $40 \mathrm{~s}$ \\
\hline $\mathrm{Pi}_{6}$ (WG; no disintegrant) & $5.6(4.0-6.5)$ & $28 \mathrm{~s}$ \\
\hline $\mathrm{Pi}_{7}$ (WG; no disintegrant) & $5.6(5.3-7.0)$ & $33 \mathrm{~s}$ \\
\hline Reference & $3.9(3.3-4.7)$ & $3 \mathrm{~min} 19 \mathrm{~s}$ \\
\hline
\end{tabular}

Pi, pilot; $D C$, direct compression; $W G$, wet granulation; $I G$, intragranulate disintegrant; $E G$, extragranulate disintegrant.

\section{Disintegration}

Disintegration results are presented in Table 3. The batches manufactured by wet granulation presented some correlation with hardness values and disintegration times. The highest disintegration time was from the reference product.

\section{Dissolution Profile}

Comparative dissolution profiles (Figs. 1 and 2) of pilot batches and the reference product were obtained using recommended USP tests 1 and 2 and with the biorrelevant dissolution medium simulating the conditions of empty gut (pre-prandial) (Fig. 3) (11). The comparison was based on similarity values $\left(f_{1}\right.$ and $f_{2}$; simple model independent approach). In tests performed using the dissolution media recommended in USP, the lots showed more than $85 \%$ dissolution in 15 minutes; thus, $f_{1}$ and $f_{2}$ were not calculated.

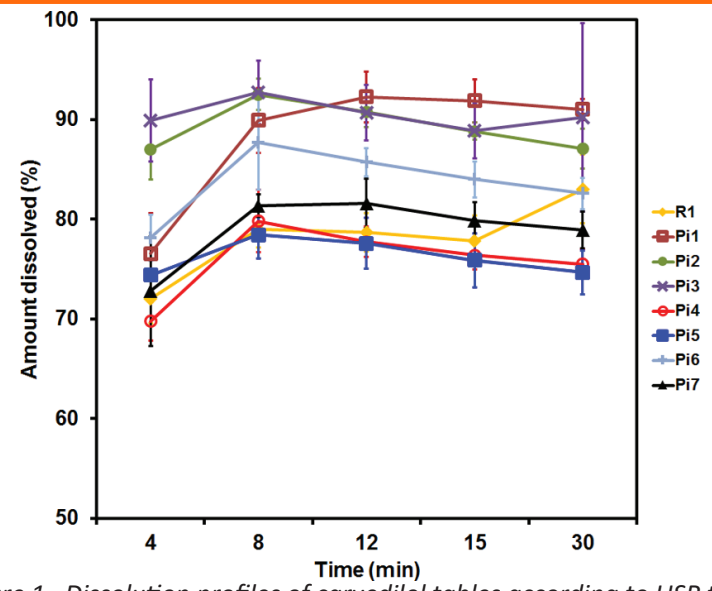

Figure 1. Dissolution profiles of carvedilol tables according to USP test 1.

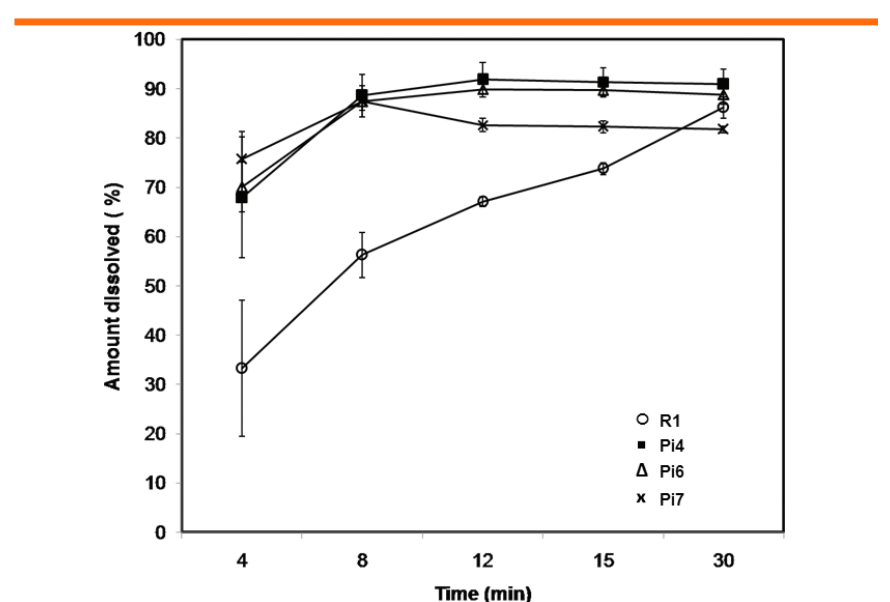

Figure 2. Dissolution profiles of carvedilol tables according to USP test 2. $R$, Reference; Pi, Pilot.

The polymorphic property of carvedilol may be related to some variations in dissolution profile. In fact, we have demonstrated before that carvedilol has different polymorphs, which can impact its dissolution behavior (9). However, in other work, it was observed that all carvedilol batches used to formulate the tablets were made of the same polymorph, so no difference observed in the dissolution profiles could be attributed to this solid state property of the raw materials (15). Moreover, no further characterization of the chirality of this API was made, but if it was different between the batches used, the main impact would be related to the polymorphism. Chirality is a characteristic attributed to the molecule that could have an impact in packing on the crystal lattice. If no difference is observed in terms of crystallinity, then it can be suggested that this property was not relevant to the differences observed in tablets dissolution.

\section{Dissolution Profiles Obtained in USP Test 2}

Dissolution profiles obtained with USP test 2 are presented in Figure 1. The pilot batch $\mathrm{Pi}_{1}\left(f_{2}: 48.17\right)$ presented release results higher than those of the reference product; thus the formulation of $\mathrm{Pi}_{2}$ was altered with introduction of partially pregelatinized corn starch, $50 \%$ reduction in the concentration of crospovidone and on the concentration of lactose spray dried. With the poor results with $\mathrm{Pi}_{2}\left(f_{2}\right.$ : 46.37), the formulation via wet granulation was initiated.

Thus, batch $\mathrm{Pi}_{3}$ was produced using the disintegrant $(25 \%$ as intragranulate and $75 \%$ as extragranulate) and reducing the proportion of high water-soluble of excipients in the formulation. However, $\mathrm{Pi}_{3}$ did not achieved a significant reduction in release of carvedilol $\left(f_{2}: 44.47\right)$.

Batches $\mathrm{Pi}_{4}$ and $\mathrm{Pi}_{5}$ were manufactured with higher PVP concentrations, varying the presence of disintegrant. $f_{2}$ results were good: 71.32 for both batches. Batches $\mathrm{Pi}_{6}$ $\left(f_{2}: 56.91\right)$ and $\mathrm{Pi}_{7}\left(f_{2}: 77.30\right)$ were also approved, with 
$\mathrm{Pi}_{7}$ being the most similar to the reference product. There was also a good correlation with the release rate, hardness, and disintegration of the tablets.

Evaluating the results obtained in the sampling time set for the dissolution profile, one observes a slight decrease in the release of the drug throughout the test. This occurred in all batches using USP test 2 and was less pronounced in the reference product.

Carvedilol is a weak base and therefore would be expected that the solubility was greatest at acidic $\mathrm{pH}$ (16). However, according Chakaraborty et al., at $\mathrm{pH}$ values between 1 and 4 , the solubility of carvedilol is limited by the protonated form or by forming carvedilol hydrochloride in situ (17). According to the authors, carvedilol hydrochloride is formed in the dissolution medium such as the gastric fluid and has solubility lower than that of the base.

As mentioned, USP test 2 dissolution medium is the simulated gastric fluid without enzymes. Thus, reduced dissolution throughout the test may be related to carvedilol hydrochloride formation, but this needs to be verified by characterization of the waste deposited in the vessel after the dissolution profile tests in this medium. In the results obtained with USP test 1 , for which the dissolution medium is $\mathrm{HCl} \mathrm{pH} \mathrm{1.45,} \mathrm{reduced} \mathrm{release} \mathrm{over}$ the time was also observed.

\section{Dissolution Profiles Obtained in USP Test 1}

As shown in Figure 2, USP test 1 batches had a fast dissolution in the first minutes. The results for the first three points of the dissolution profile for the reference product are lower than those observed in test 2. However, the dissolution values for the pilot batches are higher than the values found in test 1 .

Unlike the results obtained with USP test 2, dissolution results from test 1 did correlate with hardness and disintegration results. The pilot batch using manufacturer $\mathrm{B}$ (sample with larger particle size) showed the lowest drug dissolution. However, it is not possible to demonstrate a correlation between the results of particle size and drug release for manufacturers $\mathrm{A}$ and $\mathrm{C}$ (batches $\mathrm{Pi}_{4}$ and $\mathrm{Pi}_{7}$ ).

For all samples evaluated with USP test 2, dissolution was lower with test 1 . However, the comparison is somewhat compromised, because a drop in dissolution was observed during the assay when performed by test 2 .

\section{Dissolution Profiles Obtained in USP Test 3: Fasted-State Simulated Intestinal Fluid (FaSSIF)}

Similarity values $\left(f_{1}\right.$ and $\left.f_{2}\right)$ obtained from the comparison of the dissolution profiles for the reference product and formulations using FaSSIF as medium are as follows: $\mathrm{Pi}_{4}$ (105.27 and 20.66, respectively), $\mathrm{Pi}_{6}$ (7.08 and 74.78), $\mathrm{Pi}_{7}$ (42.34 and 40.24). In this test, only the pilot batch $\mathrm{Pi}_{6}$ presented $f_{1}$ and $f_{2}$ within the recommended range. The dissolution profiles in FaSSIF are presented in Figure 3.

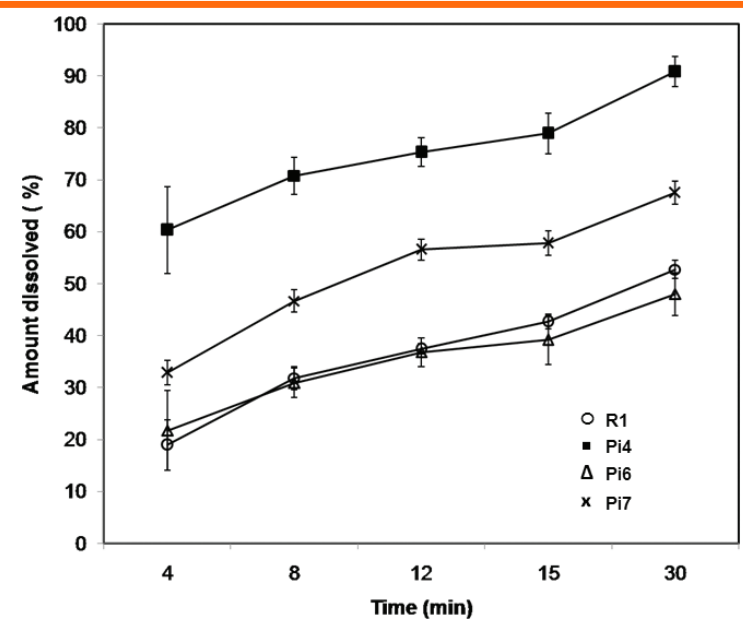

Figure 3. Dissolution profiles of carvedilol tables with Test 3 (FaSSIF). $R$, Reference; Pi, Pilot, FaSSIF, fasted-state simulated intestinal fluid.

In fasted-stated simulated intestinal fluid (FaSSIF) medium, no correlation was observed with hardness and disintegration. However, some correlation between dissolution and particle size distribution was observed. So, in this case, the bigger the particle, the lower the dissolution.

In this condition, a decline in release over time was not observed, as was seen with USP tests 1 and 2 . This result confirms the hypothesis that the acidic medium induces the generation of carvedilol hydrochloride, of lower solubility, as mentioned above.

According to Marques et al., regarding the development of formulations, the use of complex composition media that simulate the conditions of biological fluids at the site where the drug will be absorbed is very important (18). These media subject dosage forms to similar conditions as found in vivo that can enable the evaluation of different formulations providing a better understanding of the drug release mechanism.

As a Class II drug, the limiting factor for bioavailability of carvedilol is dissolution (absorption control), which means that the use of a discriminating medium contributes to the prediction of in vivo behavior. In this case, an in vitroin vivo correlation can be achieved with the use of wellplanned dissolution studies. The choice of the dissolution medium is an extremely important factor (19). 


\section{CONCLUSION}

The results show that changing composition of the dissolution medium produced different dissolution profiles for the reference product and the pilot batches. This change also impacted the end result of dissolution. The results show that even if the dissolution was evaluated in single point method, which is commonly used to perform batch-to-batch quality control, it is possible to check the impact of changing the dissolution medium.

The dissolution profile in FaSSIF was able to discriminate between the three different manufacturers, indicating that particle size has an impact on the drug release.

This is a very important assessment, keeping in view the need for in vivo studies for product approval by regulatory agencies. In addition, it is, at least according to the literature, this is the first mention of this specific evaluation in relation to carvedilol.

\section{FUNDING}

The authors disclosed no financial support related to this article.

\section{CONFLICTS OF INTEREST}

The authors disclosed no conflicts of interest related to this article.

\section{REFERENCES}

1. Lanzanova, F. A. Carvedilol - Estudo de estabilidade e perfil de dissolução. M.Sc. Dissertation, Federal University of Santa Maria, 2007.

2. Brazilian Pharmacopeia, Vol 1, 5th ed. ANVISA: Brasília, 2010.

3. Pokharkar, V. B.; Mandpe, L. P.; Padamwar, M. N.; Ambike, A. A.; Mahadik, K. R.; Paradkar, A. Development, characterization and stabilization of amorphous form of a low Tg drug. Powder Technol, 2006, 167, 20-25. DOI: 10.1016/j.powtec.2006.05.012.

4. Emshanova, S. E. Drug synthesis approaches and production technologies: methodological approaches to the selection of excipients for drug for preparation of tablets by direct pressing. Pharm. Chem. J. 2007, 42, 89-94. DOI: 10.1007/s11094-0080066-1.

5. Stojanović, J.; Vladimirov, S.; Marinković, V.; Veličković, D.; Sibinović, P. Monitoring of the photochemical stability of carvedilol and its degradation products by the RP-HPLC method. J. Serb. Chem. Soc, 2007, 72, 37-44. DOI: 10.2298/JSC0701037S.

6. Dulin, B.; Abraham, W. T. Pharmacology of carvedilol. Am. J. Cardiol. 2004, 93, 3B-6B. DOI: 10.1016/j.amjcard.2004.01.003.

7. Albers, S.; Meibohm, B.; Mir, T. S.; Lãer, S. Population pharmacokinetics and dose simulation of carvedilol in pediatric patients with congestive heart failure. Br. J. Clin. Pharmacol. 2007, 65, 511-522. DOI: 10.1111/j.1365-2125.2007.03046.x.

8. Borges, N. C. C.; Mendes, G. D.; Silva, D. O.; Rezende, V. M.;
Astigarraga, R. E.; Nucci, G. Quantification of carvedilol in human plasma by high-performance liquid chromatography coupled to electrospray tandem mass spectrometry application to bioequivalence study. J. Chromatogr. B Analyt. Technol. Biomed. Life Sci. 2005, 822, 253-262. DOI: 10.1016/j. jchromb.2005.06.012.

9. Prado, L. D.; Rrocha, H. V. A.; Resende, J. A. L. C.; Ferreira, G. B.; Teixeira, A. M. R. F. An insight into carvedilol solid forms: effect of supramolecular interactions on the dissolution profiles. CrystEngComm. 2014, 16, 3168-3179. DOI: 10.1039/ c3ce42403k.

10. Bioavailability and Bioequivalence Studies for Orally Administered Drug Products-General Considerations; Guidance for Industry; U.S. Department of Health and Human Services, Food and Drug Administration, Center for Drug Evaluation and Research (CDER), U.S. Government Printing Office: Washington, DC, 2003.

11. The United States Pharmacopeia and National Formulary USP 34-NF 29; The United States Pharmacopeial Convention, Inc.: Rockville, MD, 2011. p. 2190-2193. DOI: 10.4135/9781412963855.n1200.

12. Jantratid, E.; Janssen, N.; Reppas, C.; Dressman, J. B. Dissolution media simulating conditions in the proximal human gastrointestinal tract; an update. Pharm. Res. 2008, 25, 16631676. DOI: 10.1007/s11095-008-9569-4.

13. Dressman, J.; Kramer, J. Pharmaceutical Dissolution Testing, Taylor \& Francis Group: New York, 2005, p 419.

14. Shargel, L.; Wu-Pong, S.; Yu, A. B. C. Applied Biopharmaceutics \& Pharmacokinetics, $15^{\text {th }}$ ed.; McGraw-Hill Companies: New York, 2005, p 892.

15. Alves, J. M. V.; Prado, L. D.; Rocha, H. V. A. Evaluation and correlation of the physicochemical properties of carvedilol. Pharm. Dev. Technol. 2016, 1, 1-11. DOI: 10.3109/10837450.2015.1073740.

16. Kostewicz, E.S.;Brauns, U.; Becker, R.; Dressman, J. B. Forecasting the oral absorption behavior of poorly soluble weak bases using solubility and dissolution studies in biorelevant media. Pharm. Res. 2002, 19, 345-349. DOI: 10.1023/A:1014407421366.

17. Chakaraborty, S.; Shukla, D.; Jain, A.; Mishra, B.; Singh, S. Assessment of solubilization characteristics of different surfactants of carvedilol phosphate as a function of $\mathrm{pH}$. J. Colloid Interface Sci. 2009, 335, 242-249. DOI: 10.1016/j. jcis.2009.03.047.

18. Marques, M. R. C.; Loebenberg, R.; Almukainzi, M. Simulated biological fluids with possible application in dissolution testing. Dissolution Technol. 2011, 18, 15-28. DOI: 10.14227/ DT180311P15.

19. Waterbeemd,H.V.;Testa,B.,Eds.Drug Bioavailability-Estimation of Solubility, Permeability, Absorption and Bioavailability, Vol. 40, 2nd ed.; Wiley-VCH Verlag GmbH \& Co. KGaA: Weinheim, Germany, 2008, p 624. DOI: 10.1002/9783527623860. 